\title{
Dextrins from Maize Starch as Substances Activating the Growth of Bacteroidetes and Actinobacteria Simultaneously Inhibiting the Growth of Firmicutes, Responsible for the Occurrence of Obesity
}

\author{
Renata Barczynska $^{1}$ - Janusz Kapusniak ${ }^{1}$ Mieczyslaw Litwin ${ }^{2}$ - Katarzyna Slizewska ${ }^{3}$. \\ Mieczyslaw Szalecki ${ }^{2,4}$
}

Published online: 7 May 2016

(C) The Author(s) 2016. This article is published with open access at Springerlink.com

\begin{abstract}
Unarguably, diet has a significant impact on human intestinal microbiota. The role of prebiotics as substances supporting the maintenance of appropriate body weight and reducing the demand for energy via stimulation of the growth of beneficial microbiota of the gut and formation products such as short-chain fatty acids, is more and more often highlighted. The objective of this study was to evaluate whether dextrins from maize starch resistant to enzymatic digestion stimulate the growth of Bacteroidetes and Actinobacteria strains representing a majority of the population of colon microbiota in lean individuals and limit the growth of Firmicutes bacterial strains representing a majority of the population of colon microbiota in obese individuals. The study was conducted with the use of in vitro method, using isolates from faeces of children characterized by normal weight, overweight and obesity. It was demonstrated that dextrins from maize starch equally efficient stimulate the growth of the isolates derived from normal-weight, overweight and
\end{abstract}

Electronic supplementary material The online version of this article (doi:10.1007/s11130-016-0542-9) contains supplementary material, which is available to authorized users.

Renata Barczynska

r.barczynska@gmail.com

1 Institute of Chemistry, Environmental Protection and Biotechnology, Jan Dlugosz University in Czestochowa, Armii Krajowej 13/15, 42-200 Czestochowa, Poland

2 The Children's Memorial Health Institute, Warsaw, Poland

3 Institute of Fermentation Technology and Microbiology, Faculty of Biotechnology and Food Sciences, Technical University of Lodz, Lodz, Poland

4 Faculty of Health Sciences, UJK, Kielce, Poland obese children, and therefore may be added to foods as a beneficial component stimulating growth of strains belonging to Actinobacteria and Bacteroidetes for both overweight, obese and normal-weight children.

Keywords Dextrin $\cdot$ Prebiotics $\cdot$ Microbiota $\cdot$ Firmicutes . Bacteroidetes
Abbreviations
BCFA Branched-chain fatty acids
DRCM Differential reinforced clostridial broth
GLP-1 Glucagon-like peptide-1
HPLC High-performance liquid chromatography
IOTF International Obesity Task Force
K1 K2, dextrins (dietary fiber) preparations made from maize starch (citric acid in the K1 fiber preparation and tartaric acid in the $\mathrm{K} 2$ fiber preparation)
NBW Normal body weight
PYY Neuropeptide anorexigenic
RCA Reinforced clostridial agar
SCFA Short-chain fatty acids

\section{Introduction}

According to Roberfroid et al. 2010 [1] prebiotics are defined as "the selective stimulation of growth and/or activity(ies) of one or a limited number of microbial genus(era)/species in the gut microbiota that confer(s) health benefits to the host". Efficacy of prebiotics is owed to the fact that they are not hydrolyzed and absorbed in the upper sections of the gastrointestinal tract, so they reach the colon in uncharged form where they constitute substrates for beneficial bacteria [2]. 
More and more often, the role of prebiotics as substances supporting maintenance of appropriate body weight and reducing the demand for energy, through stimulating the growth of beneficial intestinal microbiota and the formation of such products as short-chain fatty acids (SCFA), is highlighted. Many studies indicate that obesity has a multifactorial background (genetic, environmental, social), and one of such factors are changes in the composition and metabolic function of the intestinal microbiota. It is acknowledged that it is particularly important to maintain the right proportion of Bacteroidetes and Firmicutes strains [3-7].

Studies conducted by the group of Ley, Backhed and De Filippo also indicate that obesity in humans is associated with the composition of intestinal microbiota [3, 8, 9]. Backhed et al. [8, 10] determined the contribution of Bacteroidetes and Firmicutes in obese and normal-weight mice and found that the proportion of Bacteroidetes is significantly lower in obese mice (20\%), while in normal-weight mice, the contribution of these bacteria was higher and accounted for up to $40 \%$. In turn, Fleissner et al. [11] demonstrated that diet rich in animal fats and low content of fiber administered to mice results in a reduction of the quantity of Bacteroidetes strains and the growth of Firmicutes. Moreover, studies aimed at explanation whether the use of prebiotics may promote a feeling of satiety and reduction of hunger were conducted $[12,13]$. It was demonstrated that one can associate the positive effect of prebiotics with modulation of intestinal microbiota, particularly with the production of SCFA and increased level of PYY (neuropeptide anorexigenic, this peptide is synthesized and secreted by the L-cells of the ileum and colon exhibiting stimulating effect on satiety center) and GLP-1 (glucagon-like peptide-1) resulting in reduced glycemia, insulin resistance and body fat, increasing the feeling of satiety [14-16].

The objective of the study was to evaluate whether resistant dextrins from maize starch obtained with the use of citric or tartaric acids are capable of stimulating the growth of Bacteroidetes and Actinobacteria strains representing the majority of the colon microbiota in lean individuals and at the same time reducing the growth of Firmicutes bacteria representing a majority of the colon microbiota in obese individuals.

\section{Materials and Methods}

Bacteria In the study, Lactobacillus, Bifidobacterium, Prevotella, Clostridium, Bacteroides intestinal bacteria were isolated from faeces of 20 normal-weight children and 20 overweight and obese children - patients from the Institute "Monument-Children's Health Center" in Warsaw. Selection of the study group was based on the criteria of the
International Obesity Task Force (IOTF) developed by Cole et al. [17].

Starch Formulations Dextrin was prepared at the Institute of Chemistry, Environmental Protection and Biotechnology, Jan Dlugosz University in Częstochowa. Dextrins were obtained from maize starch subjected to piroconversion and chemical modification: citric dextrin (K1) produced as a result of maize starch heating at $130{ }^{\circ} \mathrm{C}$ for $180 \mathrm{~min}$ with the addition of hydrochloric acid as a catalyst and citric acid as crosslinking agent; tartaric dextrin (K2) obtained as a result of maize starch heating at $130^{\circ} \mathrm{C}$ for $180 \mathrm{~min}$ with the addition of hydrochloric acid as a catalyst and tartaric acid as crosslinking agent.

Determination of Quantity of Bacteria Cultivation of the joint cultures of Lactobacillus, Bifidobacterium, Bacteroides, Clostridium, Prevotella intestinal bacteria isolated from faeces of 20 normal-weight children and 20 overweight and obese children, was carried out in media containing dextrins from maize starch. Prepared a suspension of bacteria that the number of cells of particular bacteria was established at about 107$108 \mathrm{cfu} / \mathrm{ml}$, the number of cells of particular bacteria was established at about $10^{7}-10^{8} \mathrm{cfu} / \mathrm{m}$ (colony forming units/ $\mathrm{m})$, which corresponds to the number of cells of these microorganisms in the initial section of the colon. Biomass bacteria was transferred into $100 \mathrm{ml}$ medium [18] containing K1 or K2 dextrin as the sole carbon source. The cultures were incubated at $37{ }^{\circ} \mathrm{C}$ for $168 \mathrm{~h}$. Immediately after inoculation, and after $24 \mathrm{~h}$ of incubation, cultivation according to Koch's plate method was carried out from each bacterial culture using the appropriate selective media. Clostridium strains were cultured with the use of differential reinforced clostridial broth (DRCM, MERCK, Darmstadt, Germany), Bacteroides strains with the use of Schaedler medium with gentamycin antibiotic (BioMerieux, Marcy I'Etoile, France), Prevotella strains using Brucella medium composed of antibiotics such as Polymyxin B, Bacitracin, Cycloheximide, Nalidixic acid, Nystatin, Vancomycin (BioMaxima, Poland), reinforced clostridial agar (RCA) [19] with the addition of dicloxacillin antibiotic was used for Bifidobacterium, Lactobacillus strains were cultured using Rogosa medium (MERCK, Darmstadt, Germany).

Determination of Lactic Acid, SCFA and BCFA Using HPLC Lactic acid, short-chain fatty acids (acetic, propionic, butyric, formic, and valeric) and branched fatty acids (BCFA; isovaleric and isobutyric) were determined by highperformance liquid chromatography (HPLC) using a Surveyor chromatograph (Thermo Scientific) and an Aminex HPX-87H column $(300 \times 7.8 \mathrm{~mm})$ from Bio-Rad Aminex ${ }^{\circledR}$ with sulfonated divinyl benzene-styrene copolymer support. The following analytical parameters were used: $300 \times 7.8 \mathrm{~mm}$ Aminex HPX-87H column, mobile phase $0.005 \mathrm{M} \mathrm{H}_{2} \mathrm{SO}_{4}, 210 \mathrm{~nm} \mathrm{UV}$ detector, injector valve with a 
sample loop, injection volume $10 \mu$ l, column temperature $60{ }^{\circ} \mathrm{C}$, flow rate $0.6 \mu \mathrm{l} / \mathrm{min}$, analysis of a single sample $35 \mathrm{~min}$. Samples with known concentrations of the acids (0; $0.125 ; 0.25 ; 0.50 ; 0.75$ and $1 \% \mathrm{acid} / \mathrm{ml}$ ) were analyzed with HPLC in order to obtain calibration curves showing acid concentration to peak area ratios.

Statistical Analysis The results were evaluated with WShapiro Wilk test assessing normality of the distribution of the results. Due to the deviation from the normal distribution, further analysis was based on U Mann-Whitney test. Statistical significance was established at $p<0.05$. The statistical analysis was performed using STATISTICA 10.0 software (StatSoft, Inc.).

\section{Results and Discussion}

The highest efficiency of stimulation of Bifidobacterium and Lactobacillus strains isolated from both, faeces of overweight and obese children ( 8.54 and $8.32 \log \mathrm{cfu} / \mathrm{ml}$, respectively) as well as from faeces of normal-weight children (8.48 and $8.28 \mathrm{log} \mathrm{cfu} / \mathrm{ml}$, respectively), was observed for dextrin obtained from maize starch with the use of citric acid (K1). The number of Prevotella and Clostridium strains isolated from faeces of overweight and obese children was comparable and accounted for 7.85 and $7.97 \mathrm{log} \mathrm{cfu} / \mathrm{ml}$ respectively, while for normal-weight children it was estimated at 8.01 and $8.20 \log \mathrm{cfu} / \mathrm{ml}$, respectively. Bacteroides strains isolated from faeces of children with overweight, obesity and normal weight used K1 dextrin with the lowest efficacy. However, the quantity of strains isolated from faeces of normal-weight children was slightly higher and was $7.94 \mathrm{log} \mathrm{cfu} / \mathrm{ml}$ in comparison to the culture of strains isolated from faeces of overweight and obese children (7.62 log cfu/ml) (Fig. 1).

Dextrin obtained from maize starch with the use of tartaric acid (K2) best stimulated the growth of Bifidobacterium strains isolated from faeces of overweight and obese children, the quantity of these strains after 24th hour of incubation was $8.53 \mathrm{log} \mathrm{cfu} / \mathrm{ml}$. Bifidobacterium strains isolated from faeces of normal-weight children and Lactobacillus strains isolated from faeces of normal-weight, overweight and obese children equally well used $\mathrm{K} 2$ dextrin as a carbon source, their number was very similar and accounted for 8.4-8.42 $\log \mathrm{cfu} / \mathrm{ml}$. The number of Prevotella strains isolated from both groups of children in the culture supplemented with $\mathrm{K} 2$ dextrin was similar and accounted for 7.83 and $7.90 \mathrm{log} \mathrm{cfu} / \mathrm{ml}$, similarly as Clostridium strains after 24th hour incubation with K2 dextrin, as their quantity was estimated at 8.03 and $8.13 \log \mathrm{cfu} /$ $\mathrm{ml}$. In analogy to the culture supplemented with $\mathrm{K} 1$ dextrin, Bacteroides strains isolated from faeces of overweight, obese or normal-weight children used K2 dextrin with the least efficiency, the quantity of these strains after 24th hour of incubation was 7.58 and $7.88 \log \mathrm{cfu} / \mathrm{ml}$, respectively (Fig. 2).

\section{The Percentage Contribution of Bacteria Belonging to Firmicutes, Bacteroidetes and Actinobacteria Types}

The percentage composition of the tested types of Firmicutes, Bacteroidetes and Actinobacteria bacteria was very similar in media supplemented with $\mathrm{K} 1$ and $\mathrm{K} 2$ dextrins independently of the dextrin used and independently of whether the strains were isolated from faeces of normal-weight, overweight or obese children. Bacteria belonging to Firmicutes type accounted for $40-41 \%$ of the population of bacteria in culture, bacteria belonging to Bacteroidetes type accounted for 38$39 \%$ of the population, while bacteria belonging to the type Acinobacteria type accounted for 21-22\% of all strains tested (Fig 3. online resource).

\section{SCFA and BCFA in Faeces of Obese and Normal-Weight Children}

After $24 \mathrm{~h}$ of joint cultivation of bacteria isolated from faeces of obese children in the medium supplemented with dextrin (K1), lactic acid concentrations ranged from 1.61 to $6.88 \mathrm{mg} / \mathrm{g}$ of faeces $(3.55 \mathrm{mg} / \mathrm{g}$ of faeces on average). SCFA concentrations ranged from 3.63 to $8.48 \mathrm{mg} / \mathrm{g}$ of faeces $(5.60 \mathrm{mg} / \mathrm{g}$ of faeces on average) whereas BCFA concentration from 0.18 to $0.52 \mathrm{mg} / \mathrm{g}$ of faeces $(0.11 \mathrm{mg} / \mathrm{g}$ of faeces on average). The lowest quantity of valeric acid $(0.42 \mathrm{mg} / \mathrm{g}$ of faeces $)$, butyric acid $(0.52 \mathrm{mg} / \mathrm{g}$ of faeces $)$ and formic acid $(0.88 \mathrm{mg} / \mathrm{g}$ of faeces) was found in culture of strains isolated from faeces of obese children containing $\mathrm{K} 1$ dextrin. The average amount of acetic and propionic acid was established at 2.65 and $1.13 \mathrm{mg} / \mathrm{g}$ of faeces. Among BCFA acids, isobutyric acid whose concentration was $0.11 \mathrm{mg} / \mathrm{g}$ of faeces was predominant, whereas the concentration of isovaleric acid was lesser and accounted for $0.04 \mathrm{mg} / \mathrm{g}$ of faeces on average. In the culture of the same strains but with the addition of dextrin (K2), lactic acid concentrations ranged from 2.72 to $4.96 \mathrm{mg} / \mathrm{g}$ of faeces (average concentration of acid was $3.75 \mathrm{mg} / \mathrm{g}$ of faeces, similar to the amount of lactic acid in the culture with K1 dextrin). SCFA concentrations ranged from 3.88 to $8.67 \mathrm{mg} / \mathrm{g}$ of faeces $(5.88 \mathrm{mg} / \mathrm{g}$ of faeces on average), while BCFA concentration from 0.18 to $0.28 \mathrm{mg} / \mathrm{g}$ of faeces $(0.23 \mathrm{mg} / \mathrm{g}$ of faeces on average). The lowest amount of butyric $(0.39 \mathrm{mg} / \mathrm{g}$ of faeces $)$ and valerianic acid $(0.40 \mathrm{mg} / \mathrm{g}$ of faeces) was found in strains isolated from faeces of obese children in which supplementation with K2 dextrin was used. The average amount of formic and 
Fig. 1 The number of tested strains isolated from faeces of overweight, obese and normalweight children in medium supplemented with $\mathrm{K} 1$ dextrin. $N B W$ normal body weight, Average
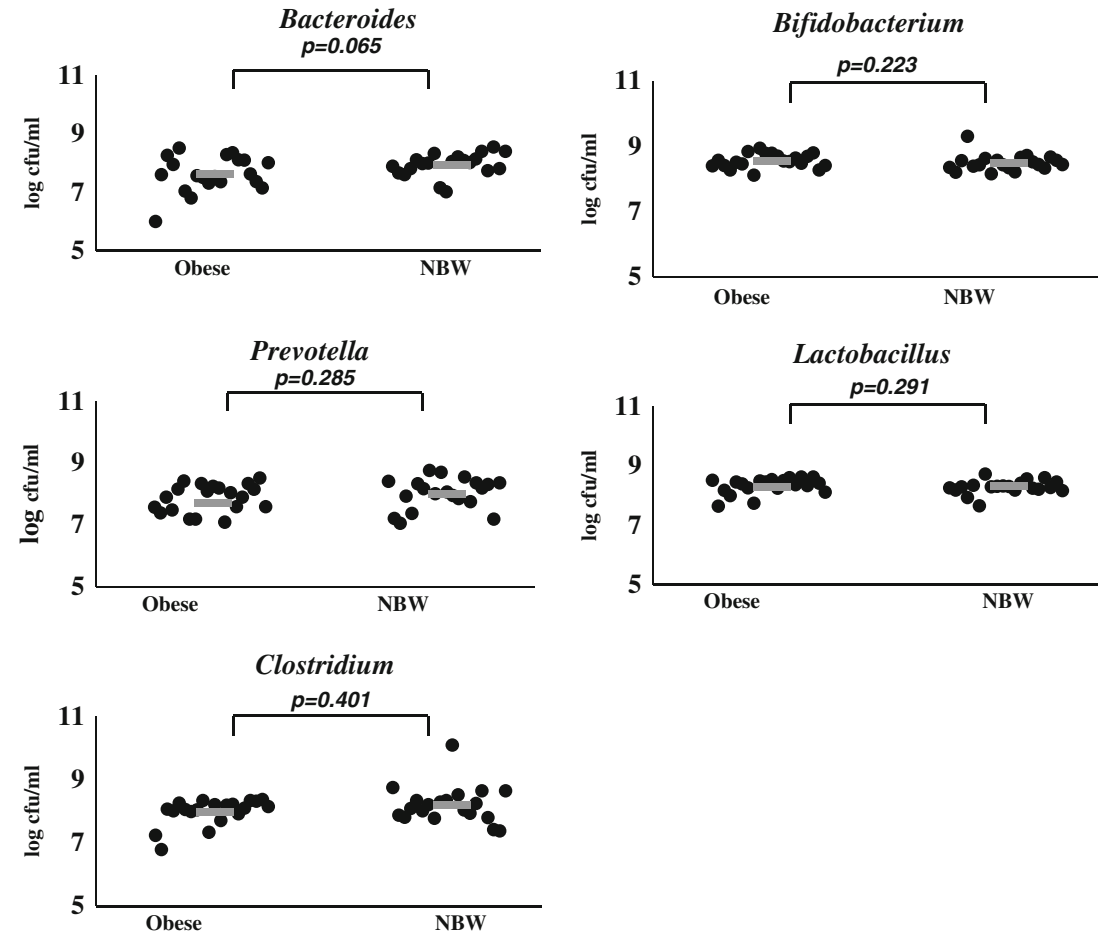

propionic acid was comparable (1.01 and $1.20 \mathrm{mg} / \mathrm{g}$ of faeces). Among SCFA, the highest amount of acetic acid $(2.86 \mathrm{mg} / \mathrm{g}$ of faeces) was found in strains isolated from faeces of obese children in which supplementation with K2 dextrin was used. Among BCFA, isobutyric acid whose concentration was $0.23 \mathrm{mg} / \mathrm{g}$ of faeces was predominant, whereas the concentration of isovaleric acid was lower and accounted for $0.02 \mathrm{mg} / \mathrm{g}$ of faeces on average. In the joint culture of bacteria isolated from faeces of obese children in the medium supplemented with $\mathrm{K} 2$ dextrin, average amount of lactic acid and SCFA was higher by about $5 \%$ in comparison to mean values for
Fig. 2 The number of tested strains isolated from faeces of overweight, obese and normalweight children in medium supplemented with K2 dextrin. $N B W$ normal body weight, Average
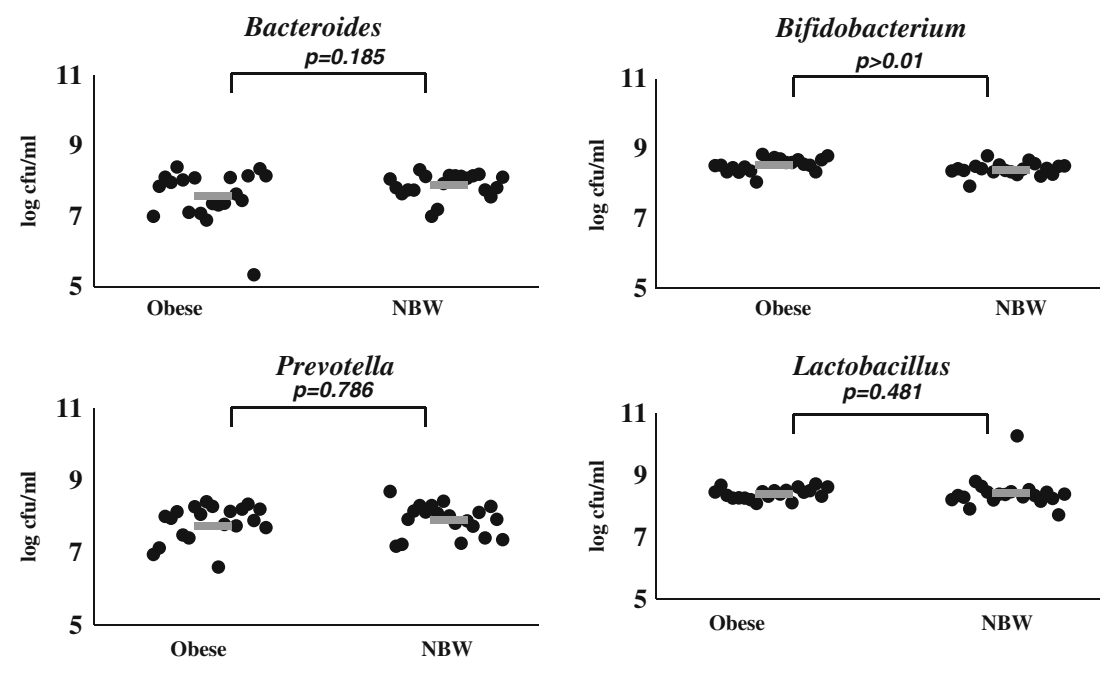

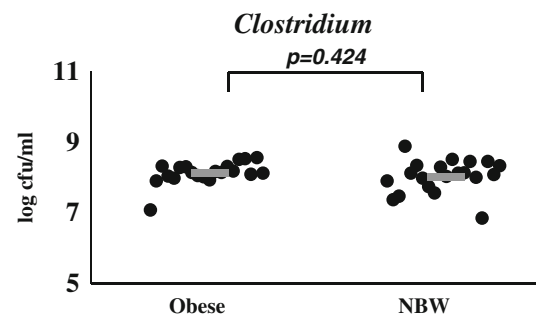


these acids in culture supplemented with $\mathrm{K} 1$ dextrin, while the average amount of BCFA was higher by about $50 \%$. After $24 \mathrm{~h}$ of joint culture of bacteria isolated from faeces of children with normal body weight in the medium supplemented with dextrin (K1), lactic acid concentration ranged from 3.06 to $9.11 \mathrm{mg} / \mathrm{g}$ of faeces $(6.04 \mathrm{mg} /$ $\mathrm{g}$ of faeces on average). SCFA concentration ranged from 4.67 to $17.00 \mathrm{mg} / \mathrm{g}$ of faeces $(9.79 \mathrm{mg} / \mathrm{g}$ of faeces on average), while BCFA concentration from 0.21 to $1.50 \mathrm{mg} / \mathrm{g}$ of faeces $(0.42 \mathrm{mg} / \mathrm{g}$ of faeces on average). The lowest amount of butyric acid $(0.80 \mathrm{mg} / \mathrm{g}$ of faeces) was found in strains isolated from faeces of children with normal body weight in which supplementation with K1 dextrin was used. The average amount of formic and pentanoic acid was similar and accounted for 1.30 and $1.46 \mathrm{mg} / \mathrm{g}$ of faeces. Among SCFA acetic $(4.19 \mathrm{mg} / \mathrm{g}$ of faeces on average) and propionic acid $(2.01 \mathrm{mg} / \mathrm{g}$ of faeces on average) were the highest in strains of culture isolated from faeces of normal-weight children in which supplementation with K1 dextrin was used. Among BCFA, isobutyric acid whose concentration was $0.41 \mathrm{mg} / \mathrm{g}$ of faeces was predominant, while the concentration of isovaleric acid was much lower and accounted for $0.04 \mathrm{mg} / \mathrm{g}$ of faeces on average. In the culture of the same strain however supplemented with K2 dextrin, lactic acid concentration, SCFA and BCFA was similar as in culture with K1 dextrin. The amount of SCFA ranged from 5.59 to $15.80 \mathrm{mg} / \mathrm{g}$ of faeces $(9.40 \mathrm{mg} / \mathrm{g}$ of faeces on average), whereas BCFA concentration from 0.21 to $1.32 \mathrm{mg} / \mathrm{g}$ of faeces $(0.50 \mathrm{mg} / \mathrm{g}$ of faeces on average). Lactic acid was predominant and its average amount was $5.67 \mathrm{mg} / \mathrm{g}$ of faeces. The lowest amount of butyric $(0.82 \mathrm{mg} / \mathrm{g}$ of faeces) and valeric $(0.91 \mathrm{mg} / \mathrm{g}$ of faeces $)$ acids was reported, while the highest concentration was found for acetic acid $(4.27 \mathrm{mg} / \mathrm{g}$ of faeces on average). The average amount of formic and propionic acids was estimated at 1.10 and $1.99 \mathrm{mg} / \mathrm{g}$ of faeces, respectively. Among BCFA, isobutyric acid whose concentration was $0.45 \mathrm{mg} / \mathrm{g}$ of faeces was predominant, whereas the concentration of isovaleric acid was much lower $(0.02 \mathrm{mg} / \mathrm{g}$ of faeces on average). In the joint culture of bacteria isolated from faeces of children with normal body weight in the medium supplemented with $\mathrm{K} 1$ dextrin, average amount of lactic acid was decreased by about $6 \%$ in comparison to the average amount of these acids in culture with $\mathrm{K} 2$ dextrin, while the amount of SCFA was decreased by $4 \%$. The average amount of BCFA in medium with the addition of $\mathrm{K} 1$ dextrin was decreased by about $16 \%$ compared to the culture with $\mathrm{K} 2$ dextrin. It was observed that the average amount of lactic acid in the culture with isolates from children with normal body weight was higher by $41 \%$ (medium supplemented with $\mathrm{K} 1$ dextrin) and $34 \%$ (medium supplemented with $\mathrm{K} 2$ dextrin) compared to the concentration of this acid in the culture of isolates from children with overweight and obesity. Similar relationship was observed for SCFA, their concentration was higher by $43 \%$ (K1) and $39 \%(\mathrm{~K} 2)$ in cultures of isolates from children with normal body weight in comparison to the isolates from obese children, also BCFA concentration was higher by $74 \%$ (K1) and $54 \%$ (K2) (Tab 1. online resource).

In the studies, one used dextrins derived from maize starch, in which one demonstrated the occurrence of approximately $70 \%$ of resistant fraction. Enhanced resistance to enzymatic digestion was caused by the presence of bonds other than typical for starch, particularly $\alpha-(1 \rightarrow 4)$-glycosidic bonds [20]. The product obtained with newly formed bonds was not metabolized in the upper section of the digestive tract, but was transferred into the colon, where constituted the source of carbon and energy for the intestinal bacteria. This fact has been investigated and dextrins were used as a carbon source in the culture medium of bacteria isolated from faeces of normal-weight, overweight and obese children. It was shown that all of the tested bacterial strains isolated from obese, overweight and lean children used resistant dextrins as a carbon source, however in different degrees. Resistant dextrins from maize starch were used by Lactobacillus and Bifidobacterium strains with the highest efficiency, while the lowest efficiency was reported for Clostridium and Bacteroides, which is consistent with previously published studies [21, 22].

De Filippo et al. [9] were demonstrated that, irrespective of the diet used, Actinobacteria, Bacteroidetes and Firmicutes types of bacteria were dominant in the digestive tract, however their proportions were different and dependent on the diet. In children from Burkina Faso, Actinobacteria and Bacteroidetes were predominant and accounted for 10.1 and $73 \%$ respectively, while Firmicutes bacteria accounted for $10 \%$. In children from Florence, one found higher body weight and higher incidence of obesity and intestinal microbial system was different in comparison to children from agricultural lands in Africa. It has been shown that Firmicutes bacteria were predominant (51\%), while Actinobacteria and Bacteroidetes, accounted for 6.7 and $27 \%$, respectively. In our studies, we demonstrated that the use of dextrins from maize starch obtained in the presence of citric acid (K1) and tartaric acid (K2) during joint cultivation of Bifidobacterium, Lactobacillus, Prevotella, Clostridium, Bacteroides strains stimulates the growth of strains belonging Actinobacteria and Bacteroidetes types (59$60 \%$ ), while stimulation to a lesser extent was observed for Firmicutes (40-41\%). Regardless whether the strains were isolated from faeces of normal-weight, overweight or obese children. 
In human organism, SCFA are produced as a result of anaerobic decomposition of dietary fiber and starch, so they are products of fermentation conducted by anaerobic bacteria colonizing cecum and colon. Due to the important role of SCFA in the human organism and the possible impact on the development of obesity, it is important that the addition of dextrins does not change the normal SCFA profile. SCFA exhibit many beneficial functions. Butyric acid stimulates the growth of intestinal epithelial tissue, nourishes intestinal cells, affects their proper maturation and differentiation. Propionic acid has a positive effect on the growth of hepatocytes, whereas acetic acid on the development of peripheral tissues. SCFA regulate glucose and lipid metabolism, stimulate proliferation and differentiation of intestinal enterocytes, contribute to a decrease in $\mathrm{pH}$ of the intestinal contents and thereby promote the absorption of minerals by increasing their solubility [23, 24].

Resistant dextrins from maize starch were metabolized via lactic, butyric, propionic and mixed fermentation. Dextrin from maize starch (K1 and K2) similarly stimulated the growth of bacteria of Actinobacteria, Bacteroidetes and Firmicutes types, while the concentration of metabolites was dependent on the type of bacteria, their origins and the dextrin used. More SCFA have been detected in culture of isolates derived from children with normal body weight in comparison to children with obesity and overweight. It should, however, be noted that obese children excrete far fewer compounds that may constitute a source of energy compared to individuals with normal weight [25]. Despite non-digestible carbohydrates, fermentation by colonic microbiota may also be attributed to proteins and amino acids, resulting in the formation of SCFA or BCFA, to which isobutyric isovaleric acids belong to. Phenols, indoles and ammonia are also products which are toxic for human organism, indicating this type of fermentation as harmful $[26,27]$. In my research as a consequence of fermentation of dextrins from maize starch, one reported very low concentration of BCFA, from 0.20 to $0.71 \mathrm{mg} / \mathrm{g}$ of faeces on average higher in cultures of isolates from children with normal body weight and lower in cultures of isolates derived from children with overweight and obesity.

\section{Conclusions}

Dextrins obtained by heating maize starch at $130{ }^{\circ} \mathrm{C}$ for 180 min with the addition of hydrochloric acid as a catalyst and citric (K1) or tartaric acid (K2) as crosslinking agents equally well stimulate the growth of the isolates derived from normal-weight, overweight and obese children and resistant dextrin stimulated an increase in the concentration of SCFA and thus may be added to foods as beneficial (health- promoting) component stimulating growth of strains belonging to Actinobacteria and Bacteroidetes types for children with overweight and obesity and for normal weight children as well. Randomised controlled clinical study is needed to confirm dextrins efficacy as a health promoting components.

Acknowledgments This project was funded by the National Science Centre allocated on the basis of the decision number DEC-2011/03/D/ NZ9/03601.

\section{Compliance with Ethical Standards}

Conflict of Interest We have no conflict of interest to declare.

Open Access This article is distributed under the terms of the Creative Commons Attribution 4.0 International License (http:// creativecommons.org/licenses/by/4.0/), which permits unrestricted use, distribution, and reproduction in any medium, provided you give appropriate credit to the original author(s) and the source, provide a link to the Creative Commons license, and indicate if changes were made.

\section{References}

1. Roberfroid M, Gibson GR, Hoyles L et al (2010) Prebiotic effects: metabolic and health benefits. Br J Nutr 2:S1-S63

2. Roberfroid MB (2002) Global view on functional foods: European perspectives. Br J Nutr 88(2):133-138

3. Ley RE, Turnbaugh P, Klein S et al (2006) Human gut microbes associated with obesity. Nature 444:1022-1023

4. Sanz Y, Santacruz A (2008) Evidence on the role of gut microbes in obesity. Rev Esp Obes 6:256-263. doi:10.1155/2008/829101

5. Bartolomeo F, Van den Ende W (2015) Fructose and fructans: opposite effects on health? Plant Foods Hum Nutr 70(3):227-237

6. Zuleta A, Sarchi MI, Rio ME et al (2004) Fermented milk-starch and milk-inulin products as vehicles for lactic acid bacteria. Plant Foods Hum Nutr 59(1):155-160

7. Rendón-Huerta JA, Juárez-Flores B, Pinos-Rodríguez JM et al (2012) Effects of different sources of fructans on body weight, blood metabolites and fecal bacteria in normal and obese nondiabetic and diabetic rats. Plant Foods Hum Nutr 67(1):64-70

8. Backhed F, Ding H, Wang T et al (2004) The gut microbiota as an environmental factor that regulates fat storage. Proc Natl Acad Sci 10:15718-15723

9. De Filippo C, Cavalieri D, Di Paola M et al (2010) Impact of diet in shaping gut microbiota revealed by a comparative study in children from Europe and rural Africa. Proc Natl Acad Sci 107: 14694-14696

10. Backhed F, Manchester JK, Semenkovich CF et al (2007) Mechanism underlying the resistance to diet-included in germfree mice. Proc Natl Acad Sci 101:15718-15723

11. Fleissner CK, Huebel N, Abd El-Bary MM et al (2010) Absence of intestinal microbiota does not protect mice from died-induced obesity. Br J Nutr 104:919-929

12. Cani PD, Amar J, Iglesias MA et al (2007) Metabolic endotoxemia initiates obesity and insulin resistance. Diabetes 56(7):1761-1772

13. Parnell JA, Reimer RA (2009) Weight loss during oligofructose supplementation is associated with decreased ghrelin and increased peptide YY in overweight and obese adults. Am J Clin Nutr 89(6): 1751-1759

14. Delzenne N, Neyrinck A, Cani PD (2011) Modulation of the gut microbiota by nutrients with prebiotic properties: consequences for 
host health in the context of obesity and metabolic syndrome. Microb Cell Factories 1:1-11. doi:10.1186/1475-2859-10-S1-S10

15. Alvarez-Castro P, Pena L, Cordido F (2012) Ghrelin in obesity, physiological and pharmacological considerations. Mini-Rev Med Chem 13(4):541-552

16. Parnell JA, Raman M, Rioux KP et al (2012) The potential role of prebiotic fibre for treatment and management of non-alcoholic fatty liver disease and associated obesity and insulin resistance. Liver Int 32(5):701-711

17. Cole TJ, Lobstein T (2012) Extended international (IOTF) body mass index cut-offs for thinness, overweight and obesity. Pediatr Obes 7(4):284-294

18. Wynne AG, McCartney AL, Brostoff J et al (2004) An in vitro assessment of the effects of broad-spectrum antibiotics on the human gut microflora and concomitant isolation of a Lactobacillus plantarum with anti-Candida activities. Anaerobe 10:165-169

19. Darukaradhya J, Phillips M, Kailasapathy K (2006) Selective enumeration of Lactobacillus acidophilus, Bifidobacterium spp., starter lactic acid bacteria and non-starter lactic acid bacteria from Cheddar cheese. Int Dairy J 16(5):439-445

20. Kapuśniak J, Jay-lin J (2007) Preparation and characteristics of enzyme-resistant pyrodextrins from corn starch. Pol J Food Nutr Sci 57:261-265
21. Barczynska R, Jochym K, Slizewska K et al (2010) The effect of citric acid modified enzyme-resistant dextrin on growth and metabolism of selected strains of probiotic and other intestinal bacteria. J Funct Foods 2:126-133

22. Barczynska R, Slizewska K, Jochym K et al (2012) The tartaric acid-modified enzyme-resistant dextrin from potato starch as potential prebiotic. J Funct Foods 4:954-962

23. Lin HV, Frassetto A, Kowalik EJ et al (2012) Butyrate and propionate protect against diet-induced obesity and regulate gut hormones via free fatty acid receptor 3-independent mechanisms. PLoS One 7:35240. doi:10.1371/journal.pone.0035240

24. Blaut M, Clavel T (2007) Metabolic diversity of the intestinal microbiota: implications for health and disease. J Nutr 137: 751-755

25. Diamant M, Blaak EE, de Vos WM (2011) Do nutrient-gutmicrobiota interaction play a role in human obesity, insulin resistance and type 2 diabetes? Obes Rev 12:272-281

26. Havenaar R (2011) Intestinal health functions of colonic microbial metabolites: a review. Benefic Microbes 2:103-114

27. Nowak A, Libudzisz Z (2006) Influence of phenol, p-cresol and indole on growth and survival of intestinal lactic acid bacteria. Anaerobe 12(2):80-84 